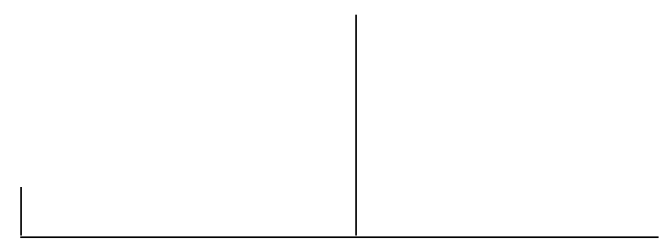

Rev. Latinoam. Psicopat. Fund., São Paulo, v. 14, n. 4, p. 690-698, dezembro 2011

\title{
Os experimentos da Guatemala: infecção intencional de presos, soldados e insanos pela Laboratório de Pesquisa em Doneça Venérea dos EUA
}

Mônica Teixeira

Em setembro, a Comissão Presidencial para o Estudo de Temas de Bioetica divulgou o relatório Ethically Impossible, sobre experimentos com seres humanos levados a cabo por médicos ligados ao serviço público de saúde dos EUA na Guatemala de 1946 a 1948, para testes relacionados a sífilis e blenorragia. Este artigo relata brevemente os acontecimentos de então e traduz parte do relatório, aquela que relata os experimentos realizados no Hospital Psiquiátrico Nacional.

Palavras-chave: DSTs., Tuskegee, consentimento informado, pesquisa com seres humanos 
Nas primeiras linhas do artigo de 2001 que inaugurou esta seção da $R e$ vista Latinoamericana de Psicopatologia Fundamental, ${ }^{1}$ os editores da seção se perguntavam o que, afinal, pode levar um médico - alguém pertencente a uma profissão cujo princípio básico e milenar é primus non nocere, antes de mais nada, não prejudicar - a insistir no caminho contrário quando se trata de experimentar novos tratamentos em pessoas. Na época, e debruçados sobre a morte de um jovem de 18 anos em consequência da inoculação de células modificadas - a chamada terapia gênica -, uma morte quase anunciada em cartas trocadas com o FDA [Food and Drug Administration, a agência que fiscaliza a comercialização de medicamentos nos EUA], a questão e as respostas (impossíveis) transitavam principalmente no campo dos motivos que poderiam mover um pesquisador tão longe do primum non nocere.

A leitura do relatório Eticamente Impossível - Pesquisa em Doenças Sexualmente Transmissíveis na Guatemala de 1946 a 1948, divulgado em setembro de 2011, e preparado a pedido de Barack Obama pela Comissão Presidencial para o Estudo de Temas da Bioética, traz de volta a questão sobre a violação do primus non nocere que, como há dez anos, permanece ainda uma vez irrespondida por Observando a Medicina. Melhor assim: a resposta é a desgraça da questão. O relato da Comissão, indignado, e construído a partir das palavras dos médicos responsáveis pela pesquisa, faz pensar, primum, sobre a crueldade dos seres humanos. Mas não apenas.

\section{Os acontecimentos}

A pesquisa sobre a qual Eticamente Impossível se debruça ocorreu por iniciativa de médicos do Laboratório de Pesquisa sobre Doenças Venéreas ("VDRL", nos documentos) da Divisão de Doença Venérea (VDD) do Serviço Público de Saúde (PHS) do governo dos Estados Unidos. O financiamento se deu por meio de um grant do então National Institute of Health, o mesmo National Institute of Health que, hoje, é a maior agência de fomen-

1. "A Indústria dos Ensaios Clínicos e sua repercussão para a prática médica contemporânea". Revista Latinoamericana de Psicopatologia Fundamental, v. 4, n. 3, p. 125, mar.2011. 


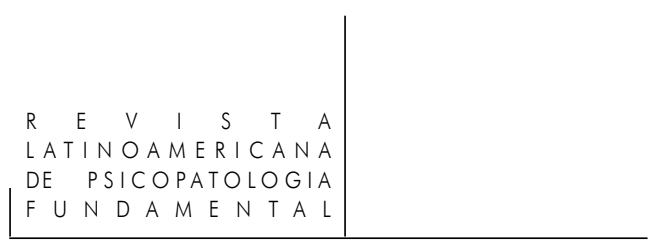

to da pesquisa em biomedicina do mundo; e foi patrocinada pelo Escritório Sanitário Panamericano, da Organização Panamericana de Saúde. O Laboratório tornou-se, mais tarde, parte do Center for Disease Control (CDC).

Em campo - quer dizer, em vários pontos do território da Guatemala -, a pesquisa contou com cooperação decidida de autoridades do governo e de médicos dos serviços de saúde locais. A estrutura montada no país, sob o comando do médico norteamericano John Cutler buscou, por um lado, identificar pessoas já infectadas com sífilis, gonorreia ou cancroide, para administrar nelas certos tratamentos e traçar a epidemiologia dessas doenças; por outro, inoculou microorganismos causadores das três doenças nas populações envolvidas nos estudos. Esse lado, da "exposição intencional" dos sujeitos de pesquisa à infecção, emerge como o mais valorizado pelos idealizadores da pesquisa na leitura da correspondência trocada entre Cutler e seus superiores e dos relatórios oficiais; e aquele que definiu como "oportunidade" a realização da pesquisa na Guatemala.

Quais guatemaltecos foram os envolvidos nas atividades do Laboratório de Pesquisa de Doença Venérea, representado ali por John Cutler? "Populações humanas vulneráveis", na definição que aparece nas primeiras linhas do memorando assinado por Barack Obama e endereçado a Amy Gutman, presidente da Comissão que elaborou Eticamente Impossível. No total, de acordo com o exaustivo levantamento da Comissão, a estrutura do Laboratório alcançou 5540 guatemaltecos - crianças em escolas, crianças em orfanatos, crianças índias e ciganas, "trabalhadoras do comércio do sexo" (prostitutas, com idade entre 16 e 18 anos), soldados, prisioneiros, pacientes de leprosário, pacientes psiquiátricos, pessoal dos EUA servindo na Guatemala e outros "não especificados". A maior parte das pessoas, em que estão incluídos todas as crianças, todas as pessoas no Leprosário, todo o pessoal dos EUA - passou apenas por testes sorológicos para diagnóstico dessas doenças sexualmente transmissíveis.

A lente da Comissão e o interesse principal dos oficiais do governo de então dos EUA recai sobre a exposição intencional às doenças em três populações, todas elas vulneráveis para a ótica de hoje e, como a comissão conclui, também para a ética de ontem e de hoje. No total, em nome especialmente do benefício que representaria para as Forças Armadas dos EUA o desenvolvimento de uma profilaxia e de um tratamento para DSTs, ${ }^{2}$ o Laboratório inoculou gonorreia em

2. Conforme citação no prefácio do relatório da Comissão Presidencial (página 12), um documento do National Research Council de 1943 informa que "aproximadamente 350 mil novas infecções com gonorreia [nas Forças Armadas] vão resultar em 7 milhões de homens/dia perdidos por ano, o equivalente a pôr fora de ação por um ano inteiro a força de (...) dez porta-aviões".

Rev. Latinoam. Psicopat. Fund., São Paulo, v. 14, n. 4, p. 690-698, dezembro 2011 
4 prostitutas, 518 soldados, 50 "insanos" (assim denominados pelo chefe do Laboratório nos EUA, e supervisor do projeto na Guatemala, John F. Mahoney, famoso por ter sido o primeiro a demonstrar a eficácia da penicilina sobre a sífilis, em 1943. Veja adiante), e em 10 sujeitos não especificados; sífilis, em 5 prostitutas, 219 prisioneiros, 446 pacientes psiquiátricos, 18 não especificados; e cancroide em 81 soldados, 41 insanos e 11 não especificados. Muitos sujeitos sofreram exposição intencional a mais de uma doença. Por exemplo, no Hospital Psiquiátrico, 486 pacientes foram envolvidos em inoculações; mas somados os números de inoculados com cada doença, o total chega a 537. Ou seja, os médicos expuseram no mínimo 51 deles a mais de uma DST.

Dos 1308 sujeitos intencionalmente expostos, 678 receberam alguma forma de tratamento. A diferença se deve principalmente a uma das principais vicissitudes enfrentadas pelo staff binacional de médicos-pesquisadores: a dificuldade em infectar alguém com gonorreia ou sífilis. Essa dificuldade abriu o caminho para a perpetração de crueldades, como se lê mais adiante.

Por que agora?

Os resultados da pesquisa (se houve) conduzida pelo laboratório na Guatemala nunca foram publicados, de acordo com o levantamento da Comissão; e o fato de sua realização sempre foi mantido velado por Cutler e outros envolvidos. Em 2003, Susan Reverby, pesquisadora em História da Medicina e professora do Wellesley College encontrou os registros das atividades nos arquivos de John Cutler, doados por ele à Universidade de Pittsburgh em 1990. Em maio de 2010, a pesquisadora apresentou suas descobertas no encontro anual da Associação Americana de História da Medicina. Ela mesma fez chegar ao Center for Diseases Control (que absorveu o Laboratório) a notícia de seu trabalho; em setembro de 2010, todo o material foi transferido para o US National Archives and Records Administration e está disponível online em http://www.archives.gov/ research/health/cdc-cutler-records/.

A revelação de que o Serviço Público de Saúde dos EUA implementou esses experimentos na Guatemala, já durante os julgamentos de Nuremberg (o primeiro deles, contra médicos por experimentação com prisioneiros, ocorreu em 1946), levou à instalação da Comissão, em dezembro de 2010; antes, em $1^{\circ}$ de outubro, o presidente Obama telefonou ao presidente guatemalteco Álvaro Colom, para apresentar desculpas oficiais dos EUA ao povo do país. Seguiu-se ao telefonema de Obama uma declaração conjunta, assinada pela Secretária da Saude, Kathleen Sibelius, e pela Secretária de Estado, Hillary Clinton. O pedido de desculpas, na 
declaração delas, se dirige especialmente aos sobreviventes e descendentes da pesquisa.

\section{Tuskegee, Alabama}

Como observa Susan Reverby, ${ }^{3}$ a revelação dos experimentos de exposição intencional de "pobres e vulneráveis" na Guatemala como que confirma o pior e inverídico rumor sobre o experimento de Tuskegee, no Alabama, em que homens negros com sífilis foram mantidos de 1932 a 1972 sem nenhum tratamento para que o mesmo Laboratório de Pesquisa de Doença Venérea pudesse "estudar" o desenvolvimento da enfermidade: o de que "secretamente" os médicos do governo teriam intencionalmente infectado os sujeitos.

Não foi assim em Tuskegee, sustenta a historiadora, mas foi sim assim na Guatemala. John Cutler, quando deixou o país, integrou-se às atividades no Alabama. Desde a revelação de que os sujeitos de pesquisa de Tuskegee não foram tratados, mesmo depois da verificação, em 1950, da eficácia da penicilina no tratamento de sífilis tardia - isto é, contraída muitos anos antes da terapia -, os acontecimentos no país da América Central vêm sendo revisados e estudados, inclusive pela professora Reverby, e poderão resultar em mudanças na política de proteção de quem participa de ensaios clínicos.

\section{"Eticamente Impossível"}

O editor de Ciências do New York Times é o autor da frase, escolhida pela Comissão para dar título ao relatório publicado em setembro de 2011. Em uma nota publicada em 27 de abril de 1947, ele escreveu:

Os doutores Harry Eagle, Harold J. Magnuson, and Ralph Fleischman, do Serviço Público de Saúde dos Estados Unidos, a Escola de Higiene John Hopkins e a Universidade da Carolina do Norte descobriram que pequenas doses de penicilina, injetadas poucos dias depois da exposição, impedem o desenvolvimento da sífilis. O caso se sustenta para coelhos, mas testes em seres humanos ainda

3. Reverby, Susan M. "Normal Exposure" and Inoculation Syphilis: a PHS “Tuskegee” Doctor in Guatemala, 1946-48. Journal of Policy History, January 2011. 
não foram realizados. Para fazer o caso para seres humanos rapidamente, seria necessário injetar neles germes vivos da sífilis, da mesma maneira com que o dr. Eagle injeta-os nos coelhos. Uma vez que isto é eticamente impossível, anos podem se passar antes que seja reunida a informação necessária.

Até o jornalista sabia que infectar pessoas com "germes vivos da sífilis", de propósito, não era possível do ponto de vista ético. A frase no New York Times, de que o doutor Cutler tomou conhecimento, ${ }^{4}$ embasa a conclusão externada pela Comissão, ao final de seu relatório, de que mesmo levando em conta os padrões de conduta em ciência praticados no meio da década de 1940, foi "moralmente errada" a conduta dos médicos do Laboratório. A instrumentalização dos sujeitos, a grave falha na obtenção do consentimento dos envolvidos, a falta de respeito na relação com eles, escreve a Comissão, não podem ser desculpados com base nas regras menos rigorosas de então.

A Comissão aponta também uma grave falha institucional - nenhum funcionário em nenhum nível hierárquico impediu a continuidade das atividades na Guatemala. O relatório evidencia que, do chefe do Conselho de Pesquisa da Presidência dos Estados Unidos, Vannevar Bush, a J.F. Mahoney, todos tomaram conhecimento, com maior ou menor detalhe, do que acontecia no país da América Central. Ninguém mandou parar antes de dois anos de atrocidade organizada.

\section{O relatório}

Observando a Medicina escolheu traduzir e publicar, na íntegra, as páginas do relatório que descrevem as atividades de exposição intencional de 446 pacientes do Hospital Psiquiátrico Nacional à sífilis. Deles, 294 foram tratados depois da exposição. A escolha baseou-se na evidente dificuldade de cumprir a regra número 1 da experimentação com seres humanos: o consentimento informado. Além disso, o relato dos procedimentos para a inoculação forçada é chocante.

4. Em carta a seu superior John F. Mahoney, de 17 de maio de 1947, Cutler cita a notícia do New York Times, mencionando a afirmação do jornalista sobre a questão ética de testar os resultados em humanos e sugere que o trabalho na Guatemala seja mantido em sigilo. A carta relata o andamento do trabalho e pode ser lida nas páginas $88-89$ do documento postado em http:// media.nara.gov/research/health/cdc-cutler-records/folder-11-cutler-1947-correspondence.pdf. Acesso em: 23 nov. 2011.

Rev. Latinoam. Psicopat. Fund., São Paulo, v. 14, n. 4, p. 690-698, dezembro 2011 


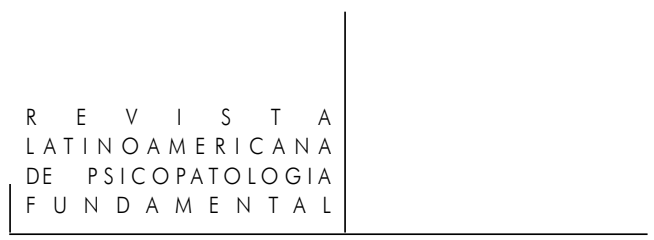

Berta: Uma história destacada pela Comissão

"Berta era paciente no Hospital Psiquiátrico. Sua idade e a doença que a levou ao hospital são desconhecidos. Em fevereiro de 1948, Berta foi injectada com sífilis em seu braço esquerdo. Um mês depois, desenvolveu sarna. Várias semanas depois, o dr. Cutler notou que ela também desenvolveu bolas vermelhas no local da injeção, lesões em seus braços e pernas, e que sua pele estava começando a se descolar de seu corpo. Berta não foi tratada da sífilis até três meses depois da infecção. Logo depois, em 23 de agosto, o dr. Cutler registrou que Berta parecia estar perto de morrer, mas não especificou o porquê. Nesse mesmo dia, ele colocou pus de gonorreia colhido de um outro paciente nos olhos de Berta, e também em sua uretra e reto. Além disso, reinfectou-a com sífilis. Alguns dias depois, os olhos de Berta estavam cheios de pus da gonorreia, e ela estava sangrando pela uretra. No dia 27 de agosto, ela morreu".

\section{As fontes}

Não relacionei cada citação a um documento em particular dos arquivos de John Cutler, como feito no texto original traduzido (http://www.bioethics.gov/ $\mathrm{cms} / \mathrm{sites} /$ default/files/Ethically-Impossible_PCSBI.pdf). Na página "Records of Dr. John F. Cutler" do National Archive dos EUA (http://www.archives.gov/ research/health/cdc-cutler-records/), fotografias também estão publicadas. De acordo com Reverby, centenas delas resultam do trabalho de Eliese Cutler, mulher do médico.

\section{O personagem principal}

John C. Cutler viveu até 2003, como um festejado especialista nas hoje "DSTs". Participou, na ordem, dos experimentos de 1943-1944 na penitenciária Terre Haute, no Estado de Indiana, em que falharam os esforços do Laboratório de Pesquisa em Doenças Venéreas para criar uma metodologia efetiva para a inoculação intencional de sujeitos com DSTs; capitaneou o projeto da Guatemala, de 1946 a 1947; em 1953, participou do experimento na Prisão Sing Sing, em que 62 prisioneiros foram submetidos a inoculação cutânea e subcutânea de contaminados com sífilis; em 1960, se juntou ao Experimento de Tuskegee, já mencionado. Em 1988, escreveu "Venereal Disease Control by Health Departaments in 
the Past: Lessons for the Present", artigo bastante conhecido publicado no American Journal of Public Health, edição de 23 de maio. ${ }^{5}$

\section{O texto traduzido}

Compreende as páginas 52 a 68 do documento Ethically Impossible. A seção de onde foi extraído o relato sobre os experimentos de inoculação intencional de insanos com a bactéria da sífilis intitula-se "Experimentos de Sífilis", subdivisão Experimentos de Exposição Intencional. Traduzi a íntegra da subseção "Hospital Psiquiátrico".

\section{Resumos}

(The Guatemala Experiments: intentional infection of prisoners, soldiers and insane patients by the United States Venereal Disease Research Laboratory)

In September 2011, the United States Presidential Commission for the Study of Bioethics released a report entitled Ethically Impossible, describing the report on human experiments conducted by physicians working at the United States Public Health Service in Guatemala, between 1946 and 1948, related to testing for syphilis and gonorrhea. This article briefly describes the events of that time and translates that part of the report which describes the experiments performed at the National Psychiatric Hospital.

Keywords: STDs. Tuskegee, informed consent, research on human subjects

(Les expériences du Guatemala: infection intentionnelle de prisonniers, soldats et malades mentaux par le Laboratoire Américain de Recherche sur les Maladies Vénériennes)

En septembre de cette année, la Commission présidentielle pour l'étude des problèmes bioéthiques a publié un rapport intitulé Ethically Impossible (éthiquement impossible) concernant des expériences sur des êtres humains menées par des médecins du service de la santé publique des États-Unis au Guatemala de 1946 à 1948 par rapport à des tests de syphilis et de gonorrhée. Cet article décrit en bref les événements de cette époque et traduit la partie du rapport qui décrit les expériences réalisées à l'Hôpital Psychiatrique National du Guatemala.

Mots clés: MST, Tuskegee, consentement éclairé, recherche sur des êtres humains

5. Disponível em: <http://ajph.aphapublications.org/cgi/reprint/78/4/372.pdf〉. Acesso em: 30 out. 2011.

Rev. Latinoam. Psicopat. Fund., São Paulo, v. 14, n. 4, p. 690-698, dezembro 2011 
(Los experimentos en Guatemala: infección intencional de presos, soldados y dementes por el Laboratorio de Investigación de Enfermedades Venéreas de los EUA)

En Septiembre, la Comisión Presidencial para el estudio de temas de Bioética divulgó el relatorio Ethically Impossible, acerca de experimentos con seres humanos relacionados a exámenes para sífilis y blenorrea que fueron realizados por médicos del Servicio Público de Salud de los EUA durante los años de 1946 a 1948 en Guatemala.

Este arítuclo relata brevemente los acontecimientos de esa época y traduce la parte del relatorio que se refiere a los experimentos realizados en el Hospital Psiquiátrico Nacional.

Palabras clave: DSTs, Tuskegee, consentimiento informado, investigación con seres humanos

Citação/Citation: TeIXEIRA, M. Os experimentos da Guatemala: infecção intencional de presos, soldados e insanos pelo Laboratório de Pesquisa em Doença Venérea dos EUA. Revista Latinoamericana de Psicopatologia Fundamental, São Paulo, v. 14, n. 4, p. 690-698, dez.2011.

Editor do artigo/Editor: Mônica Teixeira; Prof. Dr. Erney Plessmann de Camargo

Recebido/Received: 6.11.2011 / 11.6.2011 Aceito/Accepted: 10.11.2011/11.10.2011

Copyright: () 2009 Associação Universitária de Pesquisa em Psicopatologia Fundamental/ University Association for Research in Fundamental Psychopathology. Este é um artigo de livre acesso, que permite uso irrestrito, distribuição e reprodução em qualquer meio, desde que o autor e a fonte sejam citados/This is an open-access article, which permits unrestricted use, distribution, and reproduction in any medium, provided the original author and source are credited.

Financiamento/Funding: A autora declara não ter sido financiada ou apoiada/The author has no support or funding to report.

Conflito de interesses/Conflict of interest: A autora declara que não há conflito de interesses/The author declares that has no conflict of interest.

\section{Mônica TeIXeIra}

Jornalista; coordenadora geral do programa Universidade Virtual do Estado de São Paulo na TV Cultura (São Paulo, SP, Brasil).

e-mail: monica_teixeira@uol.com.br

Rev. Latinoam. Psicopat. Fund., São Paulo, v. 14, n. 4, p. 690-698, dezembro 2011 